\title{
Subpicosecond energy transfer from a highly intense THz pulse to water: A computational study based on the TIP4P/2005 rigid-water-molecule model
}

\author{
Pankaj Kr. Mishra, ${ }^{1,2,3, *}$ Oriol Vendrell, ${ }^{1,2, \dagger}$ and Robin Santra ${ }^{1,2,3}$ \\ ${ }^{1}$ Center for Free-Electron Laser Science, DESY, Notkestraße 85, D-22607 Hamburg, Germany \\ ${ }^{2}$ The Hamburg Centre for Ultrafast Imaging (CUI), Luruper Chaussee 149, D-22761 Hamburg, Germany \\ ${ }^{3}$ Department of Physics, University of Hamburg, Jungiusstraße 9, D-20355 Hamburg, Germany
}

(Received 1 November 2015; published 14 March 2016)

\begin{abstract}
The dynamics of ultrafast energy transfer to water clusters and to bulk water by a highly intense, subcycle $\mathrm{THz}$ pulse of duration $\approx 150 \mathrm{fs}$ is investigated in the context of force-field molecular dynamics simulations. We focus our attention on the mechanisms by which rotational and translational degrees of freedom of the water monomers gain energy from these subcycle pulses with an electric field amplitude of up to about $0.6 \mathrm{~V} / \AA$. It has been recently shown that pulses with these characteristics can be generated in the laboratory [C. Vicario, B. Monoszlai, and C. P. Hauri, Phys. Rev. Lett. 112, 213901 (2014)]. Through their permanent dipole moment, water molecules are acted upon by the electric field and forced off their preferred hydrogen-bond network conformation. This immediately sets them in motion with respect to one another as energy quickly transfers to their relative center of mass displacements. We find that, in the bulk, the operation of these mechanisms is strongly dependent on the initial temperature and density of the system. In low density systems, the equilibration between rotational and translational modes is slow due to the lack of collisions between monomers. As the initial density of the system approaches $1 \mathrm{~g} / \mathrm{cm}^{3}$, equilibration between rotational and translational modes after the pulse becomes more efficient. In turn, low temperatures hinder the direct energy transfer from the pulse to rotational motion owing to the resulting stiffness of the hydrogen bond network. For small clusters of just a few water molecules we find that fragmentation due to the interaction with the pulse is faster than equilibration between rotations and translations, meaning that the latter remain colder than the former after the pulse. In contrast, clusters with more than a few tens of water molecules already display energy gain dynamics similar to water in condensed phases owing to inertial confinement of the internal water molecules by the outer shells. In these cases, a complete equilibration becomes possible.
\end{abstract}

DOI: 10.1103/PhysRevE.93.032124

\section{INTRODUCTION}

Water in its liquid form is the universal solvent in which most chemical processes in a biological environment take place [1]. This fact alone is probably the main motivation behind the many efforts devoted to understanding the equilibrium and dynamical properties of liquid water. It is nowadays well established through experimental [2-5] and theoretical [6-10] investigations that the energy redistribution between internal vibrations and librational modes, and between localized librational excitations and monomers in the first and second solvation shells, occurs in ultrafast time scales of the order of hundreds of femtoseconds.

The reorientation dynamics of water plays a major role in the energy relaxation of librational excitations $[7,8]$. In particular, perturbation of one of the hindered rotational coordinates of a water monomer in the liquid phase is followed by energy transfer to neighboring water molecules in time scales as fast as $50 \mathrm{fs}$ [10]. A torque on the rotational motion of water molecules can be exerted by external electric fields that try to orient the water dipole along their polarization direction and perturb the equilibrium arrangement formed by the hydrogen bond (H bond) network [11,12]. For example, a dc or slowly varying electric field with an amplitude in the range of $0.5 \times 10^{7}-10 \times 10^{7} \mathrm{~V} / \mathrm{cm}$ can induce structural

\footnotetext{
*pankaj.kumar.mishra@desy.de
}

†oriol.vendrell@cfel.de transformations in water clusters and modify their equilibrium geometry from ringlike to chainlike structures [13,13-17].

Subcycle, highly intense $\mathrm{THz}$ pulses of pulse duration on the order of a few hundreds of femtoseconds oscillate much more slowly than most other time scales of the system and their interaction with water resembles the interaction of dc fields. These subcycle pulses are characterized by carrier frequencies on the order of 1-3 THz, which sets them off resonance with intramolecular degrees of freedom of the water monomers. Owing to their spectral width they cover a broad range of low energies of the vibrational spectrum of liquid water of mostly librational nature $[18,19]$.

At high intensity, subcycle $\mathrm{THz}$ pulses can transfer a large amount of energy to liquid water, which can be the equivalent of several hundred kelvins $[19,20]$. In previous studies based on $a b$ initio molecular dynamics (AIMD) simulations we investigated the interaction of liquid water [20] and liquid water solvating a molecular solute [19] with a short and very intense $\mathrm{THz}$ pulse reaching field amplitudes up to $0.6 \mathrm{~V} / \AA$. Pulses of similar peak field amplitude in the frequency range of $1-10 \mathrm{THz}$ have recently been demonstrated using nonlinear organic crystals [21]. There, we established that the interaction of this strong $\mathrm{THz}$ pulse with liquid water at initially $300 \mathrm{~K}$ transfers on average more than $1000 \mathrm{~cm}^{-1}$ of energy to each molecule in the system, which represents a temperature increase from 300 to about $900 \mathrm{~K}$ within 1 ps. The energy transfer to hindered rotational motion and center of mass translations occurs within a few tens of femtoseconds as the $\mathrm{THz}$ pulse reaches its maximum amplitude. 
The time scales for energy transfer from the $\mathrm{THz}$ pulse to hindered rotations and hindered translations and for internal equilibration of the energy transferred by the pulse agree well with the fast time scale for librational energy relaxation following a localized fluctuation out of equilibrium of one of the water molecules, as reported from nonequilibrium molecular dynamics simulations [10]. This is however different from the case of excitation by a strong $\mathrm{THz}$ pulse, where all water molecules in the system experience the strong external perturbation and each of them has suddenly highly excited neighboring monomers $[19,20]$.

In this work we address the question of how clusters of increasing size, starting from the single monomer case, interact with such very intense $\mathrm{THz}$ pulses. Since the $\mathrm{THz}$ pulse interaction leads to a very rapid temperature increase it is not clear a priori whether small clusters will gain energy through similar mechanisms as in the liquid phase, or clusters of small size will be rather torn apart by the $\mathrm{THz}$ pulse.

We also investigate the effect that different initial conditions of temperature and density have on the total energy transfer from the $\mathrm{THz}$ pulse and on the energy redistribution among the different types of degrees of freedom. The response of water clusters and condensed phases of water to such type of strong electromagnetic perturbations in the low frequency $\mathrm{THz}$ regime is hitherto unknown, and experiments are not available yet since it was only recently that generating pulses of these characteristics became possible [21].

In order to cover the wide range of cluster sizes and initial thermodynamic conditions that we are addressing, we must abandon the AIMD approach used in previous works and instead rely here on a molecular force field, in particular the TIP4P/2005 rigid-water-molecule model [22]. There are two important differences between our previous AIMD simulations and this work. First, here the water molecules are rigid, so no energy transfer to internal degrees of freedom is permitted. Second, the system cannot be polarized, either by neighboring water molecules or by the external field.

The energy increase of liquid water initially at $300 \mathrm{~K}$ and $1 \mathrm{~g} / \mathrm{cm}^{3}$ from AIMD corresponds to roughly an increase of $650 \mathrm{~K}$ when expressed as a temperature $[19,20]$. The same initial conditions simulated at the TIP4P/2005 level lead to a temperature increase of $400 \mathrm{~K}$. This difference can be attributed to the lack of polarization response in the force-field case, in which interaction with the external field occurs at the level of permanent dipoles only.

However, as discussed later in detail, the time-scale for energy transfer to hindered rotations and translations in the force-field case is qualitatively the same as obtained from AIMD simulations (cf. Fig. 1 in Ref. [19]). By comparison, about $60 \%$ to $70 \%$ of the energy gain in the AIMD case can be attributed to the interaction of the unpolarized system with the $\mathrm{THz}$ pulse.

These observations indicate that a qualitatively correct picture of the underlying mechanisms for energy transfer and energy redistribution can be obtained on the basis of force-field results. In particular, comparison between the rigid SPC-E and the polarizable AMOEBA force fields reported in Ref. [8] lead to results in qualitative agreement with each other for reorientation times of water molecules in the liquid phase. In the present case, the force-field simulations systematically underestimate the total energy transfer from the $\mathrm{THz}$ pulse and the corresponding temperature increase in the system by about $30 \%$ to $40 \%$ due to missing polarization effects.

The structure of the paper is as follows. Section II describes the computational methods. Section III A discusses the interaction of the $\mathrm{THz}$ pulse with water clusters and the transition to bulk behavior. Section III B describes the response of bulk water at different initial temperatures and densities to the $\mathrm{THz}$ pulse, and Sec. IV concludes and provides some outlook.

\section{COMPUTATIONAL DETAILS}

Simulations of the water clusters and of bulk water under periodic boundary conditions were performed with the Large-scale Atomic/Molecular Massively Parallel Simulator (LAMMPS) [23] using the rigid TIP4P/2005 water force field model [22]. For the water cluster simulations, 1000 initial atomic configurations and velocities were generated from a long molecular dynamics (MD) trajectory performed under canonical (NVT) conditions and equilibrated at $200 \mathrm{~K}$. Different sets of initial conditions were sampled with a $10 \mathrm{ps}$ time interval to avoid artificial correlations between each sampled phase space point. The Nosé-Hoover thermostat [24,25] was used to maintain the temperature for the phase space sampling NVT trajectory. Each set of 1000 initial conditions was then propagated microcanonically for $1.5 \mathrm{ps}$ in the presence of the $\mathrm{THz}$ pulse. For all trajectory propagations the velocity-Verlet algorithm was used with a time step of $1 \mathrm{fs}$. Water clusters of sizes $1,2,4,8,32$, and 64 monomers were initially considered. In order to study the effect of inertial confinement, a bigger spherical cluster with 8843 water molecules and radius $\approx 50 \AA$ was equilibrated at $300 \mathrm{~K}$ and subsequently exposed to the $\mathrm{THz}$ pulse. All water cluster simulations were performed with nonperiodic boundaries in all directions.

Bulk water simulations at different initial temperatures and densities were based on thermally equilibrated cubic boxes containing 2048 water molecules. By selecting the box size, a range of initial densities of $1.0 \mathrm{~g} / \mathrm{cm}^{3}, 0.296 \mathrm{~g} / \mathrm{cm}^{3}$, $0.064 \mathrm{~g} / \mathrm{cm}^{3}$, and $0.019 \mathrm{~g} / \mathrm{cm}^{3}$ were considered. Each box was then equilibrated at different temperatures of $200 \mathrm{~K}, 300 \mathrm{~K}$, $400 \mathrm{~K}, 500 \mathrm{~K}$, and $600 \mathrm{~K}$. A cutoff radius of $15 \AA$ was used for the calculation of the short-range Lennard-Jones interactions. Each set of initial density and temperature conditions was subsequently propagated microcanonically for $1.5 \mathrm{ps}$ in the presence of the $\mathrm{THz}$ pulse.

The same half cycle $\mathrm{THz}$ pulse profile as in previous investigations was considered here $[19,20]$. The THz pump pulse is given by

$$
\mathbf{E}(t)=\epsilon(t) \mathbf{u}_{z} \cos \left(\omega_{c} t+\phi\right)
$$

where $\epsilon(t)=A \exp \left\{-\left(t-t_{0}\right)^{2} / 2 \sigma^{2}\right\}$ is a Gaussian envelope with $\sigma=84.93 \mathrm{fs}$. This corresponds to a full width at half maximum (FWHM) of $\epsilon^{2}(t)$ of $141 \mathrm{fs}$. The maximum electric field amplitude $A=0.61 \mathrm{~V} / \AA$ corresponds to a power per unit area of $5 \times 10^{12} \mathrm{~W} / \mathrm{cm}^{2}$. The mean photon frequency considered is $\omega_{c}=2 \pi \times 3 \mathrm{THz}\left(100 \mathrm{~cm}^{-1}\right)$ which results in a pulse between a half and a full cycle long. $\mathbf{u}_{z}$ is the polarization direction of the electric field and $\phi$ is the carrier to envelope phase which is set to $\pi / 2$. By convention, the 
THz pulse envelope is centered at $t_{0}=0$ in all simulations and microcanonical trajectories start at $t=-250$ fs.

The analysis of the energy gain by water clusters and bulk water is performed on the basis of decomposing the total kinetic energy $\left(E_{K}^{(m)}\right)$ of each rigid water monomer $m$ in terms of its rotational energy $\left(E_{R}^{(m)}\right)$ and translational energy $\left(E_{T}^{(m)}\right)$ components. This is simply achieved in Cartesian coordinates by

$$
E_{T}^{(m)}=\frac{\left|\sum_{a_{m}} M_{a_{m}} \mathbf{V}_{a_{m}}\right|^{2}}{2 \sum_{a_{m}} M_{a_{m}}}
$$

and

$$
\begin{aligned}
E_{R}^{(m)} & =\sum_{a_{m}} \frac{\mathbf{j}_{a_{m}}^{2}}{2 M_{a_{m}}\left|\mathbf{x}_{a_{m}}\right|^{2}}, \\
\mathbf{j}_{a_{m}} & =M_{a_{m}}\left(\mathbf{x}_{a_{m}} \times \mathbf{v}_{a_{m}}\right),
\end{aligned}
$$

where $\mathbf{x}_{a_{m}} \equiv \mathbf{X}_{a_{m}}-\mathbf{X}_{m}$ and $\mathbf{v}_{a_{m}} \equiv \mathbf{V}_{a_{m}}-\mathbf{V}_{m}$ define the position and velocity of atom $a$ in monomer $m$ relative to the center of mass position $\mathbf{X}_{m}$ and velocity $\mathbf{V}_{m}$ of the monomer, respectively. In general, at this point the kinetic energy per monomer related to vibrational motion of the remaining $3 N-6$ intramolecular coordinates $E_{V}^{(m)}=E_{K}^{(m)}-E_{T}^{(m)}-E_{R}^{(m)}$ can be obtained. In the present case, owing to the rigid nature of the water model used, $E_{V}^{(m)}=0$. In particular, the intramolecular degrees of freedom were kept fixed in LAMMPS by the SHAKE algorithm [26]. The kinetic temperatures for each subset of coordinates are connected to the corresponding mean energies by the relation

$$
\left\langle E_{X}\right\rangle=\frac{3}{2} k_{B} T_{X}
$$

where $\mathrm{X}=\mathrm{T}$ (translation), $\mathrm{R}$ (rotations), and $\mathrm{V}$ (vibration).

\section{RESULTS AND DISCUSSION}

\section{A. Interaction of the $\mathbf{T H z}$ pulse with water clusters}

\section{Water monomer and dimer}

The effect of the THz pulse on a single water monomer and on the water dimer is analyzed first. This serves to illustrate the basic mechanism by which the $\mathrm{THz}$ pulse interacts with isolated water molecules and how, in the case of intermolecular interactions between various monomers, part of this energy can be transferred to their relative translational coordinates. Later, when analyzing the energy transfer to low density water beyond the critical point, some characteristic features of the interaction with the monomer will reappear.

The ensemble averaged translational $\left\langle E_{T}\right\rangle$ and rotational $\left\langle E_{R}\right\rangle$ energies per monomer were obtained by averaging over 1000 initial configurations and velocities. In the case of the monomer and due to the intramolecular constraints, the sampling of initial conditions generates only random orientations and angular velocities of the water molecule, the latter being compatible with a target temperature of $200 \mathrm{~K}$. In the dimer case, overall rotations of the system as well as intermolecular coordinates are sampled according to the target temperature of $200 \mathrm{~K}$. The electric field is polarized along the $z$ axis in all simulations. Due to the electroneutrality of

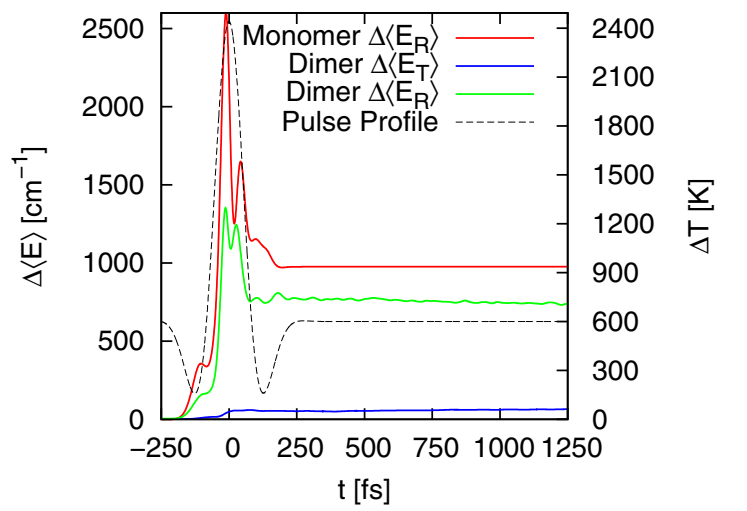

FIG. 1. Translational $\left(\Delta\left\langle E_{T}\right\rangle\right)$ and rotational $\left(\Delta\left\langle E_{R}\right\rangle\right)$ energy increase per water monomer for the monomer and dimer cases. On the right axis the kinetic temperature $T=\frac{2 E}{3 k}$ with $k$ the Boltzmann factor is shown. The THz pulse profile appears as a dotted line.

the isolated water monomer, only the rotational degrees of freedom gain energy in the presence of the $\mathrm{THz}$ pulse, as illustrated in Fig. 1. The time structure of the pulse when it changes its polarization direction can still be seen in $\Delta\left\langle E_{R}\right\rangle$ in spite of the rotational averaging over initial conditions. As expected, no further change in total energy occurs after $t=250 \mathrm{fs}$, when the pulse is over.

In the dimer case, both $\Delta\left\langle E_{R}\right\rangle$ and $\Delta\left\langle E_{T}\right\rangle$ increase due to the $\mathrm{THz}$ pulse. However, the increase of translational energy of the monomers is, in this case, much smaller than that of rotational energy. On average, the dimer fragments into its two water molecule constituents very early during or shortly after the $\mathrm{THz}$ pulse, which closes the channel for energy transfer between rotational and translational motion by interactions between the monomers. Therefore, the relative translational mode between both centers of mass remains much colder than the individual rotational modes of both monomers, and equipartition cannot be reached.

To understand the origin of the translational energy increase of the monomers we can first inspect the form of the interaction term between matter and radiation. In the nonpolarizable water model considered here the interaction of the electric field with the system is simply the sum of the interactions with the individual dipole moments of the monomers

$$
E^{w-E F}=-\sum_{m} \sum_{a_{m}} q_{a_{m}}\left(\mathbf{r}_{a_{m}} \cdot \mathbf{E}\right)=-\sum_{m} \boldsymbol{\mu}_{m} \cdot \mathbf{E},
$$

where $\mathbf{r}_{a_{m}}$ is the position of the $a$ th point charge of the $m$ th monomer, $\boldsymbol{\mu}_{m}$ is the permanent dipole moment of monomer $m$, and $\mathbf{E}$ is the external electric field. This term has no direct effect on the translational motion of the centers of mass and only depends on the orientation of each individual dipole in the electric field. The total potential energy $\left(E_{P}\right)$ of the system in the presence of the electric field consists of the sum of pair interactions between monomers and the interaction of each monomer with the field

$$
E_{P}=E_{\text {pair }}^{w-w}+E^{w-E F} .
$$

$E_{\text {pair }}^{w-w}$ includes a Lennard-Jones term between each water molecule, which is spherically symmetric, and Coulombic interactions between each effective partial charge in each water 


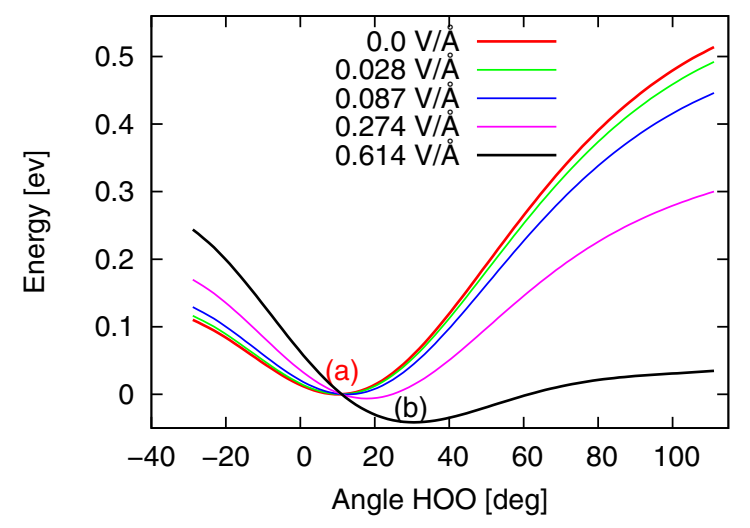

FIG. 2. Potential energy curve for HOO angle with different electric field amplitudes. The labels (a) and (b) correspond to the dimer configurations as shown in Fig. 3.

molecule, which account for the effective charge distribution in the water model [22]. This last part of $E_{\text {pair }}^{w-w}$ is strongly directional and is responsible for the adequate description of the coordination and $\mathrm{H}$ bonding between water molecules. Hence, the rotation of the water monomers forced upon them by the electric field through $E^{w-E F}$ results in unfavorable configurations due to the $E_{\text {pair }}^{w-w}$ term, which leads to translational motion of the centers of mass of the monomers and to energy transfer between monomer rotations and translations. Higher order multipolar interaction terms than dipole-dipole are embedded in the $E_{\text {pair }}^{w-w}$ term and are crucial to describe the properties of liquid water [27-30].

The effect of an electric field on the preferred relative orientation between the two water monomers is clearly seen by inspecting the potential energy curve of the water dimer along the $\angle \mathrm{HOO}$ relative angle in Fig. 2. The potential energy curves correspond to the TIP4P/2005 water force field used in the trajectory propagations. In the minimum energy configuration of the dimer, two water monomers orient themselves to form a $\mathrm{H}$ bond. The applied static electric field is polarized along the $x$ axis, which is the direction of the $\mathrm{H}$ bond in the minimum energy configuration of the dimer shown in Fig. 3(a). To construct the potential energy curves along the $\angle \mathrm{HOO}$, the donor water molecule is rotated in the $X Z$ plane around the $y$ axis. The potential energy curves were compared for static

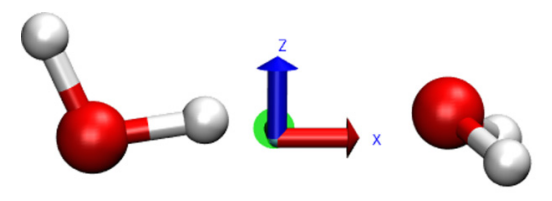

(a)

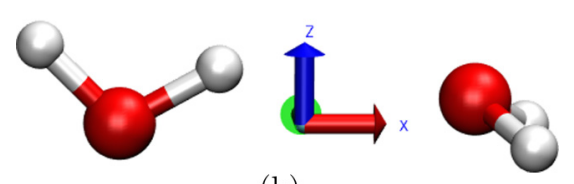

(b)

FIG. 3. Minimum energy configurations of the water dimer (a) without field and (b) with a field of $0.614 \mathrm{~V} / \AA$ as presented in Fig. 2 . electric fields with amplitudes $0,0.028,0.087,0.274$, and $0.614 \mathrm{~V} / \AA$, which in the case of a pulse would correspond to intensities of $0,10^{10}, 10^{11}, 10^{12}$, and $5 \times 10^{12} \mathrm{~W} / \mathrm{cm}^{2}$. The change in the shape of the potential is the result of the relative magnitude of the terms $E_{\text {pair }}^{w-w}$ and $E^{w-E F}$ in Eq. (6). By changing the field amplitude from $0.274 \mathrm{~V} / \AA$ to $0.614 \mathrm{~V} / \AA$, the shape of the potential energy curve changes significantly in comparison to lower amplitudes. Such high electric field is able to create a displaced and deep potential well along
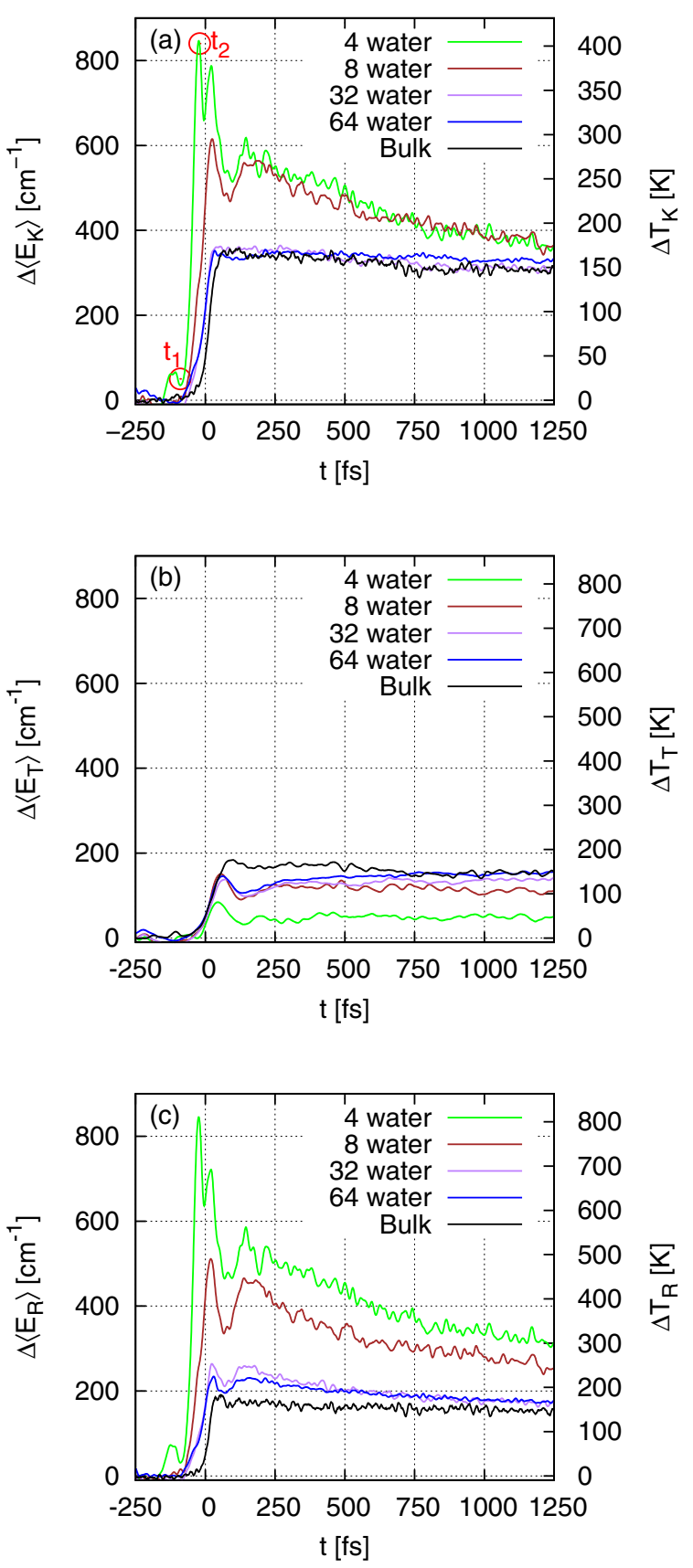

FIG. 4. (a) Ensemble averaged total kinetic energy increase $\left(\Delta\left\langle E_{K}\right\rangle\right)$, (b) translational energy increase $\left(\Delta\left\langle E_{T}\right\rangle\right)$, and (c) rotational energy increase $\left(\Delta\left\langle E_{R}\right\rangle\right)$ for the water clusters of sizes $4,8,32$, and 64 and for bulk water at $200 \mathrm{~K}$. Red circles are showing the points $\mathrm{t}_{1}$ and $t_{2}$ for 4 water cluster only. $t_{1}$ is the point when significant change happens in $\Delta\left\langle E_{K}\right\rangle$ and $\mathrm{t}_{2}$ is the point when $\Delta\left\langle E_{K}\right\rangle$ is maximum. 


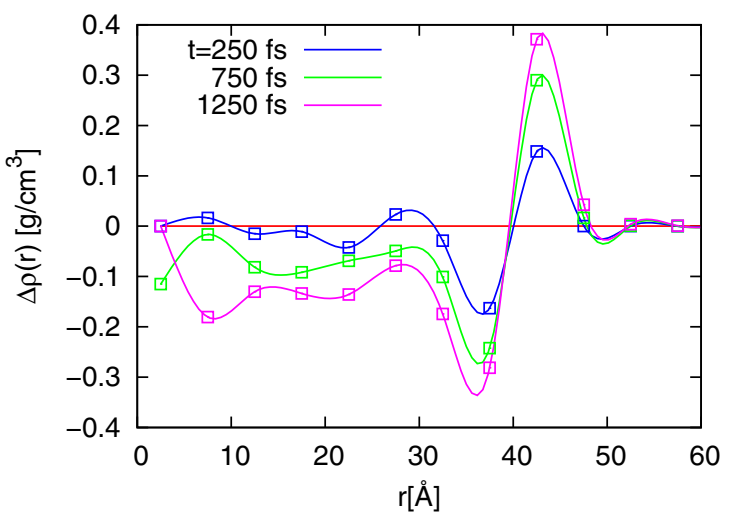

FIG. 5. Water density difference $(\Delta \rho)$ with respect to the density at $-250 \mathrm{fs}$ (before the THz pulse) at times 250, 750, and $1250 \mathrm{fs}$ in the presence of the THz pulse. $r$ is the distance to the center of the spherical cluster.

the relative orientation between the water molecules. At the high field amplitude, the electric field is strong enough to substantially alter the $\mathrm{H}$ bond and bring the water molecules out of their preferred field free relative orientation. The time delay of about $100 \mathrm{fs}$ in the rise of the translational component $\left\langle E_{T}\right\rangle$ compared to the start of the pulse and seen in Fig. 1 is related to the fact that the electric field has to reach a certain peak amplitude in order to disrupt the $\mathrm{H}$ bond and push the water molecules into configurations for which their relative distance will start to change.

The H-bond strength of the water dimer is $23.8 \mathrm{KJ} / \mathrm{mol}$ $(\approx 0.25 \mathrm{eV})$ [31]. As a consequence, a certain threshold value $\epsilon_{T}$ for the external electric field amplitude is required in order to bring water molecules out of their preferred H-bond arrangements [13,15], as already seen in Fig. 2 . The THz pulse can then be divided into different parts, depending on whether the electric field amplitude $\epsilon(t)$ is larger or smaller than the threshold value $\epsilon_{T}$ required to disrupt the H-bond structure. From Fig. 2 one can estimate $\epsilon_{T}$ to be of the order of 0.2 to $0.3 \mathrm{~V} / \AA$. Only when $\epsilon(t)>\epsilon_{T}$, which for the half cycle pulse of Eq. (1) is achieved only in the central oscillation, the electric field can effectively bring molecules out of their

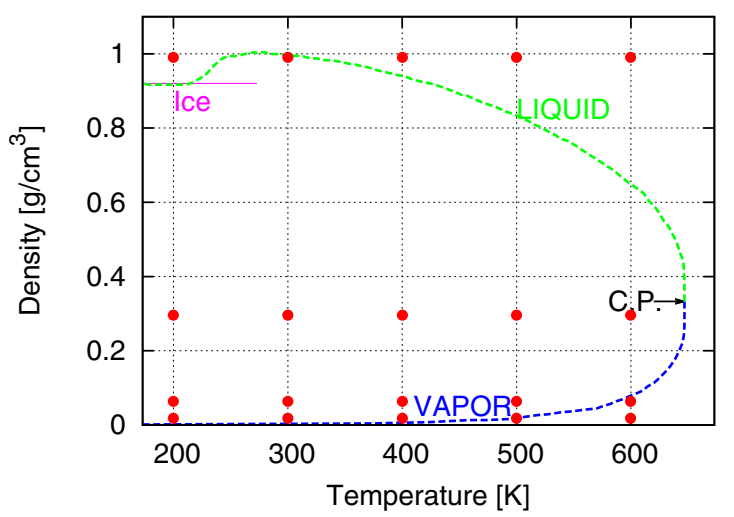

FIG. 6. Temperature $(T)$-density $(\rho)$ phase diagram of pure water [34]. C.P. is the critical point. Red dots represent the thermodynamical states of water in the phase diagram that have been taken as initial conditions to explore the effect of $\mathrm{THz}$ pulse.
H-bond arrangements. This is the reason why, already for the dimer case, $\Delta\left\langle E_{R}\right\rangle$ increases mostly during the central pulse oscillation and hardly changes during the two side wings of smaller amplitude.

\section{Water tetramer and larger clusters}

For water clusters larger than the dimer the average number of $\mathrm{H}$ bonds per water molecule increases from $1 / 2$ to between 3 and 4. As may already be anticipated, this has profound consequences for the dynamics of energy increase and energy transfer in the clusters. Figure 4 illustrates the total kinetic, rotational, and translational energy increase for water clusters of sizes $4,8,32,64$, and finally bulk water at the same initial temperature of $200 \mathrm{~K}$. This temperature was chosen to ensure equilibration of the clusters without evaporation (loss) of water molecules into their surroundings, which occurs at higher temperatures. There are various features of the energy intake dynamics that can now be understood on the basis of the observations made for the case of the water dimer. (i) Smaller clusters feature a larger energy increase immediately after the $\mathrm{THz}$ pulse because they have on average fewer $\mathrm{H}$ bonds per
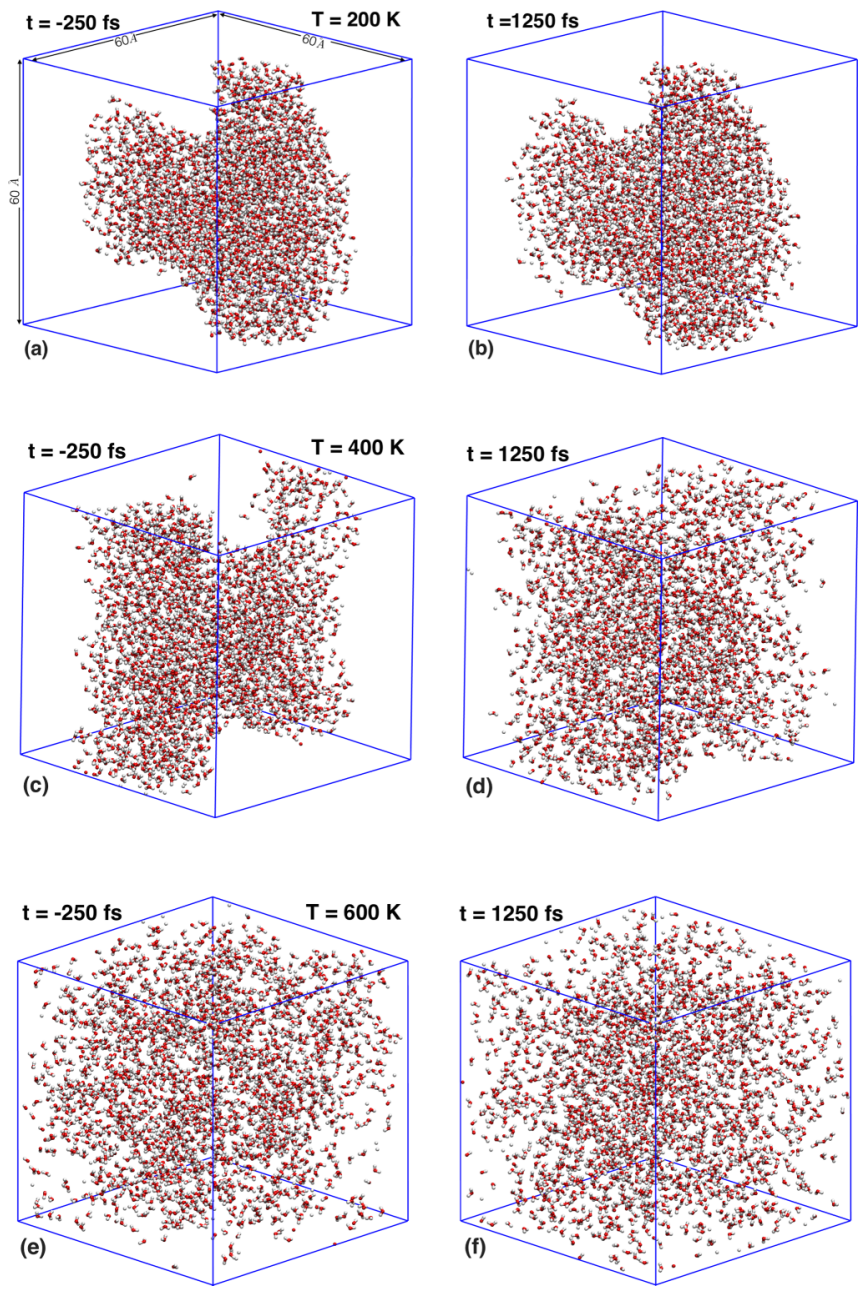

FIG. 7. Snapshots of simulation boxes with density $0.3 \mathrm{~g} / \mathrm{cm}^{3}$ and different temperatures $(T=200 \mathrm{~K}, 400 \mathrm{~K}$, and $600 \mathrm{~K})$ before the pulse, $t=-250 \mathrm{fs}$ [(a), (c), (e)], and after the pulse, $t=1250 \mathrm{fs}$ [(b), (d), (f)]. 
monomer, as seen in both Figs. 4(a) and 4(c). (ii) $\Delta\left\langle E_{R}\right\rangle$ becomes increasingly delayed as the cluster size increases because the number of surface water molecules with fewer $\mathrm{H}$ bonds decreases in relation to the cluster's volume. As a consequence, the time $t_{2}$, defined as the time of maximum energy gain, becomes increasingly delayed for larger clusters. Exactly the same trend is observed for $t_{1}$, the time at which $\Delta\left\langle E_{K}\right\rangle$ starts increasing significantly. (iii) Equilibration between the $\Delta\left\langle E_{R}\right\rangle$ and $\Delta\left\langle E_{T}\right\rangle$ components [cf. Figs. 4(b) and $4(\mathrm{c})]$ is achieved for clusters with 32 and 64 water molecules, besides the bulk system. For the smaller clusters with 4 and 8 water molecules, collisional energy transfer stops as the clusters start expanding, similarly as in the dimer case. The stability of neutral clusters at various temperatures and the applicability of statistical theories to describe the likelihood and rate of breakup have been the subject of extensive studies in the past [32]. Here we are interested in the response at
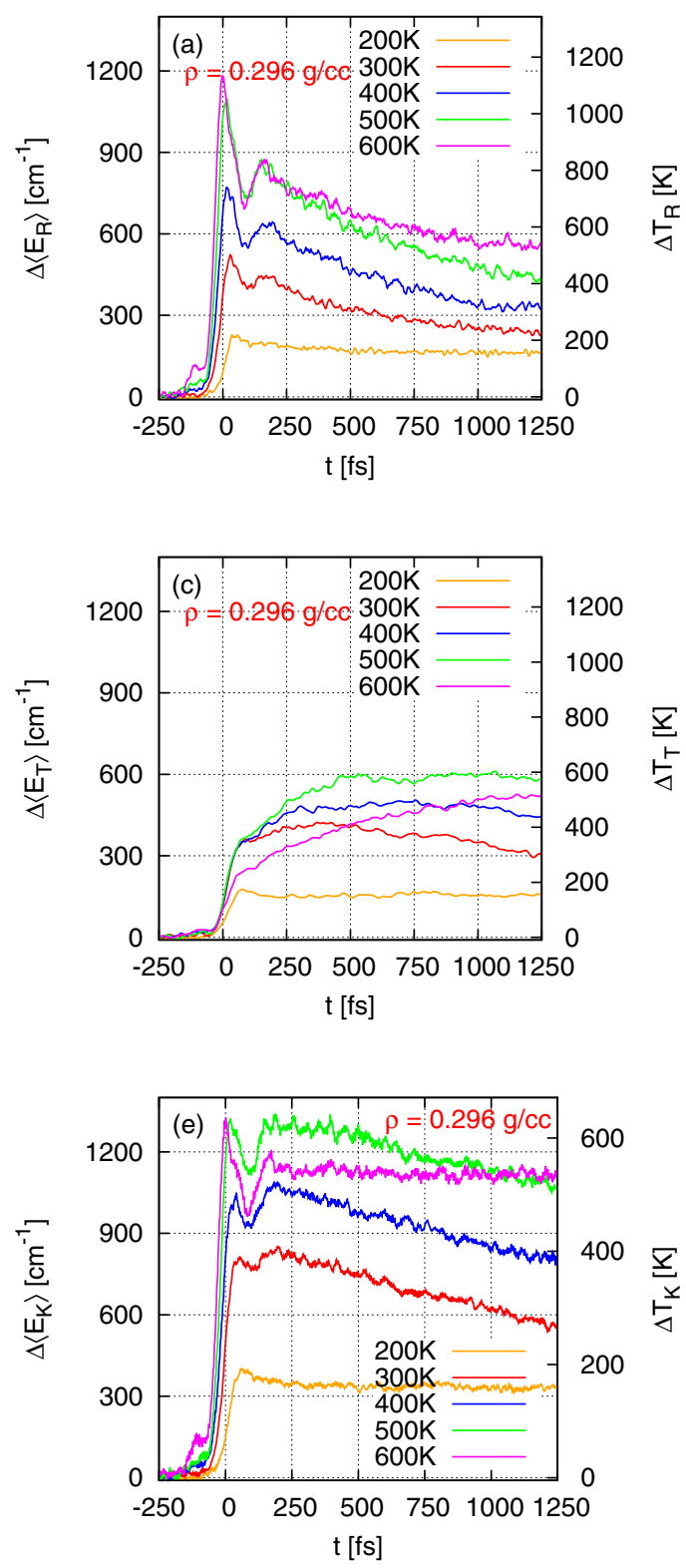

short times prior to microcanonical equilibration, which is naturally described by molecular dynamics. (iv) The total kinetic energy $\Delta\left\langle E_{K}\right\rangle$ decreases after the pulse for the smaller clusters. Since the total energy of each cluster is conserved after the pulse, the total kinetic energy decrease corresponds to an increase in the relative potential energy between monomers as the small clusters expand. This corresponds to evaporative cooling taking place at the surface of the clusters as outer water molecules leave the cluster after interaction with the THz pulse [33].

\section{Inertial confinement in ultrafast heated clusters}

In earlier investigations on liquid water interacting with the THz pulse at a density of $1 \mathrm{~g} / \mathrm{cm}^{3}$, constant volume had been assumed $[19,20]$. This rests on the assumption that the number of water molecules in the region interacting with the $\mathrm{THz}$ pulse
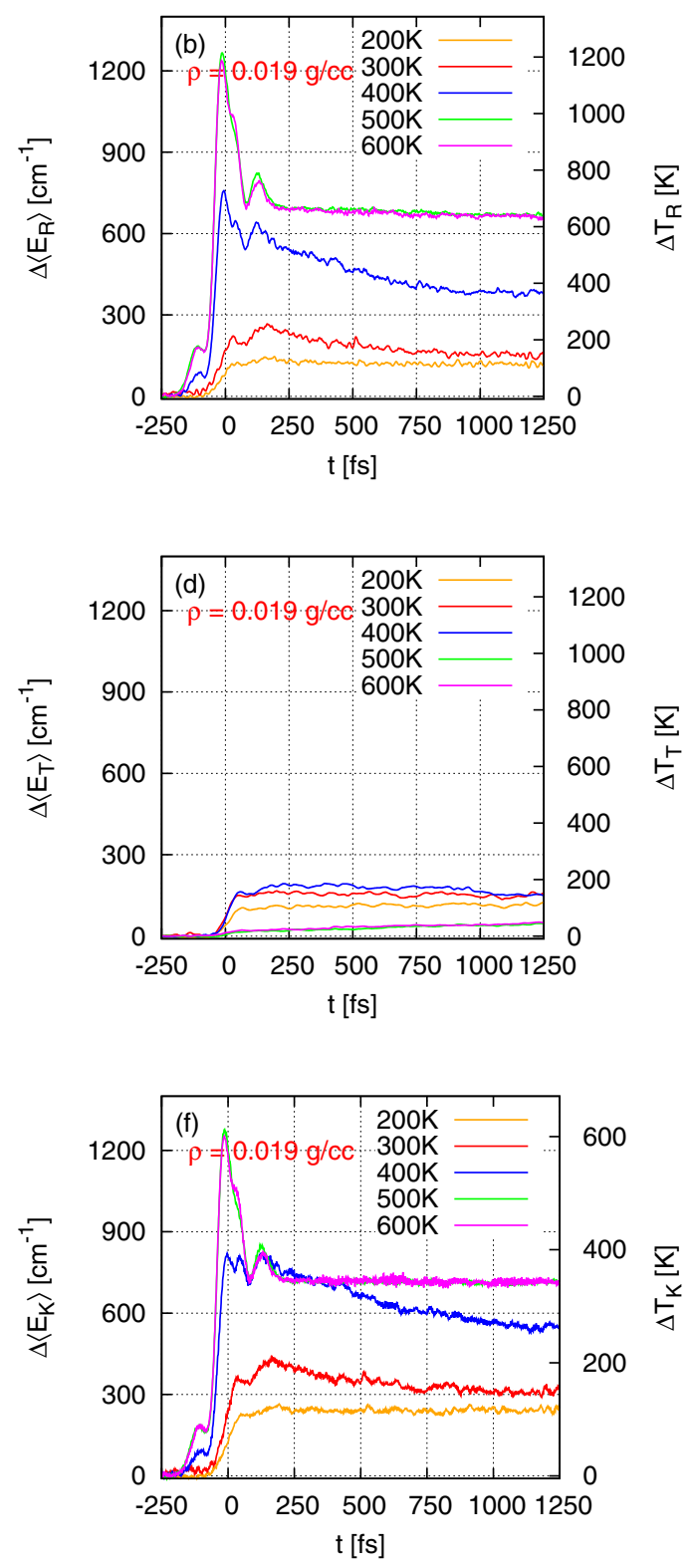

FIG. 8. Total kinetic energy $\left(\Delta\left\langle E_{K}\right\rangle\right)$, translational energy $\left(\Delta\left\langle E_{T}\right\rangle\right)$, and rotational energy $\left(\Delta\left\langle E_{R}\right\rangle\right)$ per water molecule at different temperatures for densities $0.296 \mathrm{~g} / \mathrm{cm}^{3}$ [(a), (c), (e)] and $0.019 \mathrm{~g} / \mathrm{cm}^{3}$ [(b), (d), (f)]. 
remains constant, or in other words, there is no substantial expansion of the system during and after the interaction with the $\mathrm{THz}$ pulse in the time scales of interest. As an indication that this may be the case, we already saw in the previous section that at least the total energy gain converges to the bulk value for clusters with a few tens of water molecules. Here we investigate this assumption in more detail on the basis of force-field treatment of water, such that several thousand water molecules can be taken into account. The dynamics of an isolated spherical cluster of radius $40 \AA$ and 8843 water molecules, which was extracted from a thermally equilibrated periodic box of liquid water at $300 \mathrm{~K}$, were simulated in the presence of the $\mathrm{THz}$ pulse. The initial density in the interior of the cluster was set to $1 \mathrm{~g} / \mathrm{cm}^{3}$.

The density as a function of the distance to the center of the cluster was calculated by binning the radial coordinate in spherical shells of width $5 \AA$ at times -250 (before the pulse), 250, 750, and 1250 fs relative to the center of the $\mathrm{THz}$ pulse. As seen in Fig. 5, the density varies the most in the outer 5 to $10 \AA$ shell, which diffuses out by also 5 to $10 \AA$. The density in the inner regions of the cluster remains quite stable, decreasing by about $0.15 \mathrm{~g} / \mathrm{cm}^{3}$ during the first picosecond after the pulse. Larger clusters may still lead to a more effective inertial confinements, and it appears justified to assume a constant density in simulations of heated bulk water at the time scales of interest.

\section{B. THz pulse interaction with bulk water}

We have discussed above how an intense, subcycle $\mathrm{THz}$ pulse interacts with an isolated water molecule and with water clusters of various sizes. Summarizing, an isolated water molecule interacts with the pulse through its permanent dipole moment thus gaining a large amount of rotational energy. Only when several water molecules are in close proximity can part of the rotational energy be transformed into relative translational energy owing to the coupling between rotational and relative translational motion of the water monomers in the cluster.

In the following, we explore the interaction of the THz pulse and the subsequent energy transfer dynamics in bulk water at different constant densities and equilibrated at different initial temperatures. Regarding initial temperature, this parameter is easily controllable in experimental realizations. As for density, we have already shown that, on the time scales of interest, the inner part of the cluster remains at its initial density during the interaction with the pulse and is inertially confined by the outer shell. Therefore, simulations at the constant density (i.e., constant volume) of the liquid phase, $1 \mathrm{~g} / \mathrm{cm}^{3}$, are justified. Moreover, the macroscopic density under real physical confinement of the interaction volume by, e.g., hard walls can be maintained during and after the heating-up process. Therefore, we consider various densities ranging from very low up to normal liquid density. Depending on the density and initial temperature in equilibrium, the aggregation state of water may vary from gas phase to liquid or amorphous solid phases and the coexistence of the two. This is illustrated in the saturation diagram of pure water shown in Fig. 6 [34]. In the regions inside the curve vapor and liquid water coexist. Outside the curve only one phase is present, either water vapor or condensed water. The dynamics of energy transfer from the
$\mathrm{THz}$ pulse and the subsequent energy redistribution among rotational and translational degrees of freedom are strongly dependent on the possible aggregation states found in the diagram. Those initial conditions at which simulations have been performed in this work are marked with a red dot in Fig. 6.

There are three distinctive features of the heating-up dynamics of water clusters that vary strongly as a function of the size of the cluster. First, for isolated water molecules and for clusters up to 8 monomers the amplitude profile of the $\mathrm{THz}$ pulse is imprinted in the rotational energy of the water molecules. This is indicative of none or few hydrogen bonds per monomer as compared to the bulk or to larger clusters. As a consequence, water molecules are rotationally accelerated and shortly thereafter slowed down in the presence of the $\mathrm{THz}$ field before they can have significant collisions with neighboring monomers. This can be observed in Figs. 1 and 4(c). Second, an equilibration between the rotational and translational components of the kinetic energy is only achieved for clusters after a certain minimum size. In our simulations we find equilibration between rotational and translational energy components for clusters of 32 or more water molecules. Smaller clusters
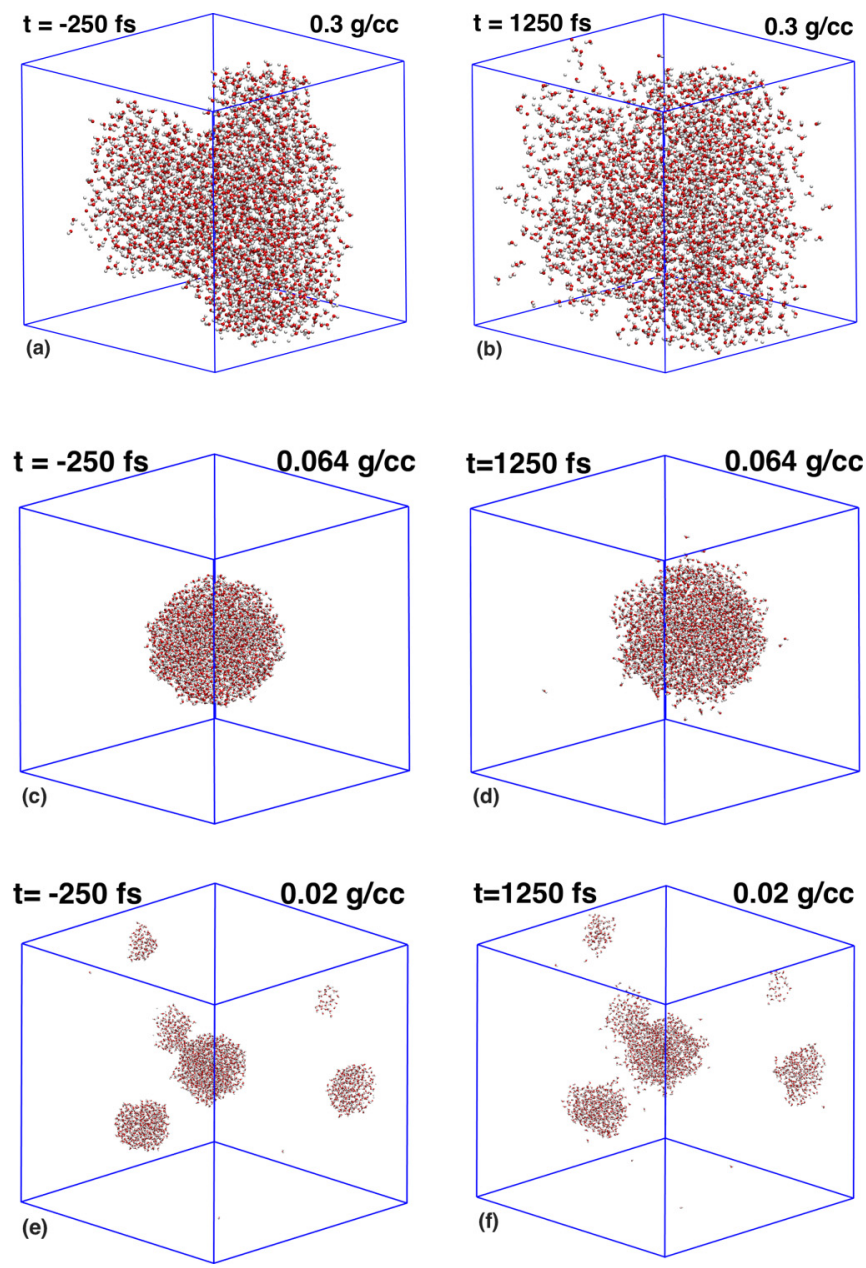

FIG. 9. Snapshots of water systems at $T=300 \mathrm{~K}$ and different densities $\left(0.3,0.064\right.$, and $\left.0.02 \mathrm{~g} / \mathrm{cm}^{3}\right)$ before the pulse, $t=-250 \mathrm{fs}$ [(a), (c), (e)], and after the pulse, $t=1250 \mathrm{fs}$ [(b), (d), (f)]. 
fragment before equilibration through collisions can complete. An extreme example of this corresponds to the water dimer and tetramer. Third, the total kinetic energy of the clusters with four and eight monomers decreases during the first picosecond as the clusters expand, which is mirrored by the corresponding increase of potential energy as the monomers separate from each other against attractive electrostatic interactions. This effect is not observed for larger aggregates, which mostly maintain their structure.

These basic features can be recognized when letting bulk water interact with the $\mathrm{THz}$ pulse at different densities and initial temperatures. Snapshots of 2048 water molecules at a density of $0.296 \mathrm{~g} / \mathrm{cm}^{3}$ for different initial temperatures of 200, 400 and $600 \mathrm{~K}$ are shown in Fig. 7. The system at $200 \mathrm{~K}$ corresponds essentially to a piece of amorphous ice with small internal energy. The system at $400 \mathrm{~K}$ is made of a large droplet of hot liquid water with smaller clusters and monomers surrounding it in the vapor state, whereas the system at $600 \mathrm{~K}$ is made of almost homogeneously distributed water molecules with a large mobility and collisional rate. This leads immediately to very different heating-up dynamics. The colder system gains in total less energy due to the more hindered water monomers. The equilibration between rotational and translational energy is however almost instantaneous due to the tight interactions between the monomers.

As the temperature increases the amplitude profile of the $\mathrm{THz}$ pulse manifests more strongly in the rotational energy component because the interactions between neighboring water molecules become progressively weaker, as seen in Fig. 8(a). Another consequence of larger temperatures is that
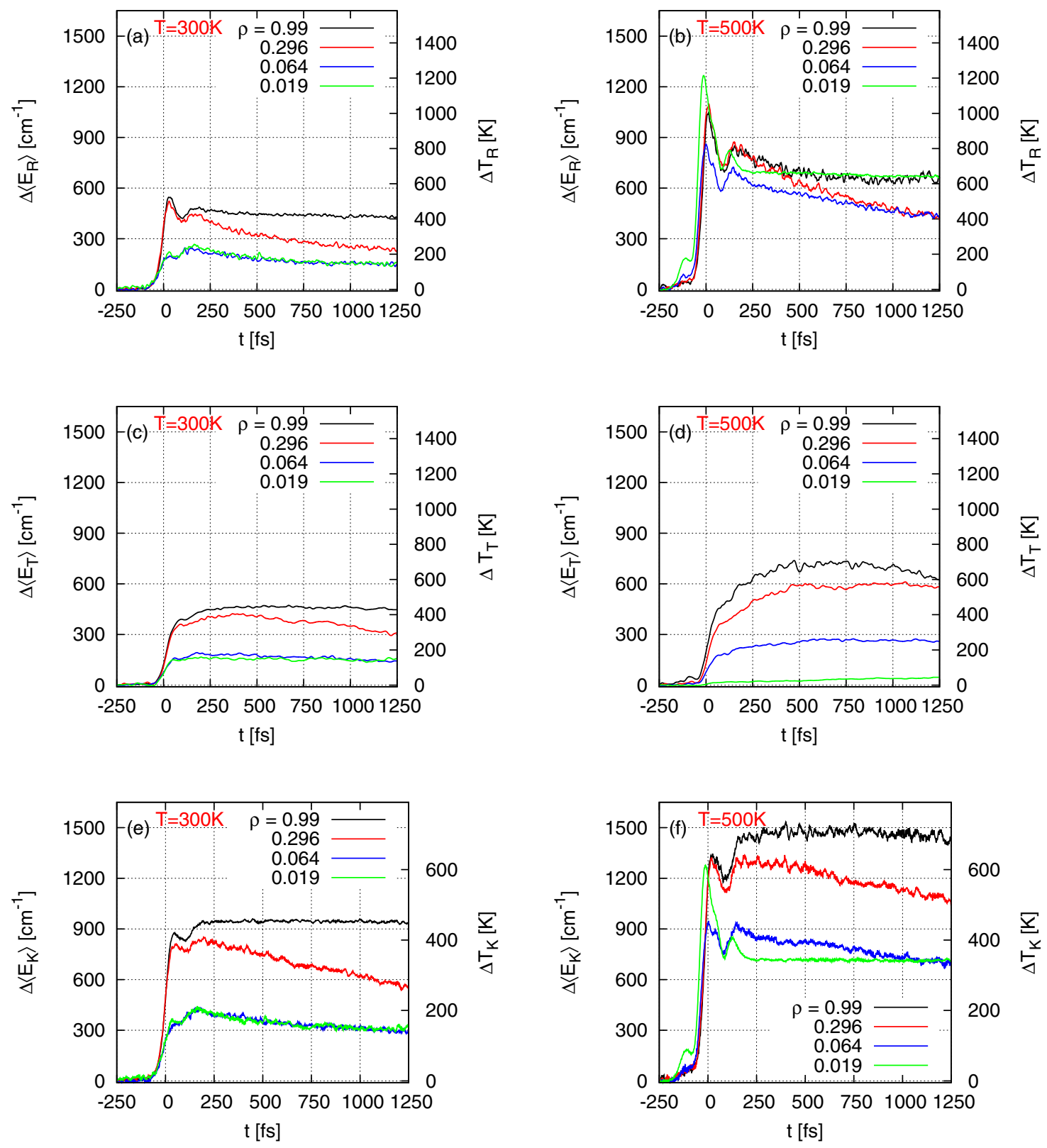

FIG. 10. Total kinetic energy $\left(\Delta\left\langle E_{K}\right\rangle\right)$, translational energy $\left(\Delta\left\langle E_{T}\right\rangle\right)$, and rotational energy $\left(\Delta\left\langle E_{R}\right\rangle\right)$ per water molecule at different densities (in $\mathrm{g} / \mathrm{cm}^{3}$ ) for initial temperatures $300 \mathrm{~K}$ [(a), (c), (e)] and $500 \mathrm{~K}$ [(b), (d), (f)] of the system. 
the energy transfer between the rotational and translational components of the kinetic energy is not instantaneous anymore as compared to colder temperatures [cf. Figs. 8(c) and 8(d)]. The slowest energy transfer to translational motion occurs at $600 \mathrm{~K}$ as a consequence of the larger separation and weaker interactions between water molecules. A decrease of the total kinetic energy shortly after the pump pulse occurs for systems initially between 300 and $500 \mathrm{~K}$ due to substantial evaporation of monomers from the outer shells of the liquid portion. This can be seen by comparing the snapshots in Figs. 9(a), 9(c), and 9(e) with those in Figs. 9(b), 9(d), and 9(f), respectively.

As compared to the previous examples, a reduced density of $0.019 \mathrm{~g} / \mathrm{cm}^{3}$ leads to differences mostly for the high temperature cases. At $T=500 \mathrm{~K}$ and $T=600 \mathrm{~K}$ the water molecules are homogeneously distributed across the available volume and at a large average distance from one another. This results in dynamics of the rotational degrees of freedom similar to those of isolated monomers and characterized by a slow rate of energy transfer to translational degrees of freedom due to infrequent collisions. This can be seen by comparing the increase of translational energy shown in Figs. 8(c) and 8(d). At this density, only the colder systems at $T=200 \mathrm{~K}$ and $T=300 \mathrm{~K}$ achieve energy equipartition between rotational and translational degrees of freedom because most of the water molecules are condensed forming ice and liquid droplets, as seen in Figs. 9(c) and 9(e). This leads to coupled hindered rotational and translational motion in the condensed phase and the corresponding fast energy transfer.

At temperatures $T=500 \mathrm{~K}$ and $T=600 \mathrm{~K}$ the amount and rate of energy transfer from the $\mathrm{THz}$ pulse to rotational motion is relatively independent of the density of the system, as can be seen in Fig. 10(b). Independently of the density, the high temperatures result in weak hydrogen bonding and the rotational degrees of freedom readily respond to the $\mathrm{THz}$ pulse. However, the collisional energy transfer from the rotational to the translational degrees of freedom at these high temperatures is strongly dependent on the density of the system, as illustrated in Fig. 10(d). At low densities almost no collisions occur whereas at $1 \mathrm{~g} / \mathrm{cm}^{3}$ the rotational and translational temperatures are equilibrated within 1 ps.

\section{CONCLUSION}

We have discussed in detail the mechanisms by which a highly intense $\left(5 \times 10^{12} \mathrm{~W} / \mathrm{cm}^{2}\right)$ and ultrashort (FWHM $141 \mathrm{fs}) \mathrm{THz}$ pulse couples to clusters of water molecules and to bulk water and transfers a large amount of energy to the system. The fundamental coupling mechanism between the $\mathrm{THz}$ pulse and the water molecules is through the permanent dipole moment of the monomers with the electric field component of the pulse. Therefore, the $\mathrm{THz}$ pulse transfers energy directly to the rotational degrees of freedom of isolated water molecules and translational motion of the center of mass remains unaffected. This changes for clusters with two or more molecules, where intermolecular coupling between hindered rotations and translations exists. Through it, the translational degrees of freedom also gain energy during and after interaction with the $\mathrm{THz}$ pulse.

We analyzed in detail the interaction between water and the $\mathrm{THz}$ pulse at different constant densities between 0.02 and $1 \mathrm{~g} / \mathrm{cm}^{3}$ and at particular initial temperatures between 200 and $600 \mathrm{~K}$. The constant density assumption (constant simulation volume) was found to be a good one since even relatively small clusters lead to inertial confinement of the water molecules already under the most external shells.

High temperatures of 500 and $600 \mathrm{~K}$ lead to rotational dynamics similar to the case of isolated water molecules. Lower temperatures, in which the interactions between water monomers are stronger, lead to a smaller energy transfer from the $\mathrm{THz}$ pulse. The density of the system plays a key role in the collisional energy transfer between rotational and translational degrees of freedom. For large temperatures, a large density leads to a high collisional rate and quick equilibration, whereas a small density prevents collisions and no equilibration can occur. For small temperatures, a large fraction of the system is found in a condensed state and equilibration between rotational and translational motion occurs almost instantaneously.

The presented results suggest that it is possible to control the total amount of energy transferred by a strong $\mathrm{THz}$ pulse and the rate at which this energy is redistributed among hindered rotations and translations by tuning the initial temperature and density of the system. This may open the door to interesting applications in which the amount and rate of energy transfer to solutes in water and could be controlled by these thermodynamic variables.

\section{ACKNOWLEDGMENTS}

We are grateful to the Virtual Institute of the Helmholtz Association "Dynamic Pathways in Multidimensional Landscapes" for financial support.
[1] J. T. Hynes, Annu. Rev. Phys. Chem. 36, 573 (1985).

[2] I. Ohmine and S. Saito, Acc. Chem. Res. 32, 741 (1999).

[3] N. Huse, S. Ashihara, E. T. Nibbering, and T. Elsaesser, Chem. Phys. Lett. 404, 389 (2005).

[4] M. L. Cowan, B. D. Bruner, N. Huse, J. R. Dwyer, B. Chugh, E. T. J. Nibbering, T. Elsaesser, and R. J. D. Miller, Nature (London) 434, 199 (2005).

[5] S. Imoto, S. S. Xantheas, and S. Saito, J. Phys. Chem. B 119, 11068 (2015).
[6] R. Rey, F. Ingrosso, T. Elsaesser, and J. T. Hynes, J. Chem. Phys. A 113, 8949 (2009).

[7] D. Laage and J. Hynes, Science 311, 832 (2006).

[8] D. Laage and J. T. Hynes, J. Phys. Chem. B 112, 14230 (2008).

[9] R. Rey and J. T. Hynes, Phys. Chem. Chem. Phys. 14, 6332 (2012).

[10] J. Petersen, K. B. Moller, R. Rey, and J. T. Hynes, J. Phys. Chem. B 117, 4541 (2013). 
[11] D. H. Jung, J. H. Yang, and M. S. Jhon, Chem. Phys. 244, 331 (1999).

[12] A. Vegiri, J. Chem. Phys. 116, 8786 (2002).

[13] Y. C. Choi, C. Pak, and K. S. Kim, J. Chem. Phys. 124, 094308 (2006).

[14] A. Vegiri and S. V. Schevkunov, J. Chem. Phys. 115, 4175 (2001).

[15] D. Rai, A. D. Kulkarni, S. P. Gejji, and R. K. Pathak, J. Chem. Phys. 128, 034310 (2008).

[16] U. Buck, I. Ettischer, M. Melzer, V. Buch, and J. Sadlej, Phys. Rev. Lett. 80, 2578 (1998).

[17] S. V. Shevkunov and A. Vegiri, J. Mol. Struct.: THEOCHEM 574, 27 (2001).

[18] M. Heyden, J. Sun, S. Funkner, G. Mathias, H. Forbert, M. Havenith, and D. Marx, Proc. Natl. Acad. Sci. USA 107, 12068 (2010).

[19] P. K. Mishra, O. Vendrell, and R. Santra, J. Phys. Chem. B 119, 8080 (2015).

[20] P. K. Mishra, O. Vendrell, and R. Santra, Angew. Chem. Int. Ed. 52, 13685 (2013).
[21] C. Vicario, B. Monoszlai, and C. P. Hauri, Phys. Rev. Lett. 112, 213901 (2014).

[22] J. L. F. Abascal and C. Vega, J. Chem. Phys. 123, 234505 (2005).

[23] S. Plimpton, J. Comput. Phys. 117, 1 (1995).

[24] D. J. Evans and B. L. Holian, J. Chem. Phys. 83, 4069 (1985).

[25] S. Nosé, Mol. Phys. 57, 187 (1986).

[26] J.-P. Ryckaert, G. Ciccotti, and H. J. Berendsen, J. Comput. Phys. 23, 327 (1977).

[27] J. L. F. Abascal and C. Vega, J. Phys. Chem. C 111, 15811 (2007).

[28] S. Niu, M.-L. Tan, and T. Ichiye, J. Chem. Phys. 134, 134501 (2011).

[29] G. N. Patey and J. P. Valleau, J. Chem. Phys. 64, 170 (1976).

[30] C. Vega and P. A. Monson, J. Chem. Phys. 102, 1361 (1995).

[31] T. Urbic, Chem. Phys. Lett. 610-611, 159 (2014).

[32] B. D. Kay and A. W. Castleman, J. Chem. Phys. 78, 4297 (1983).

[33] C. E. Klots, J. Chem. Phys. 83, 5854 (1985).

[34] W. Wagner and A. Pruß, J. Phys. Chem. Ref. Data 31, 387 (2002). 